\title{
ARID1B, a member of the human SWI/SNF chromatin remodeling complex, exhibits tumour-suppressor activities in pancreatic cancer cell lines
}

\author{
M Khursheed ${ }^{1,5}$, J N Kolla ${ }^{1,5}$, V Kotapalli ${ }^{1}$, N Gupta ${ }^{1}$, S Gowrishankar ${ }^{2}$, S G Uppin ${ }^{3}$, R A Sastry ${ }^{3,6}$, \\ S Koganti ${ }^{3}$, C Sundaram ${ }^{3}$, J R Pollack ${ }^{4}$ and M D Bashyam ${ }^{*}, 1$ \\ ${ }^{1}$ Laboratory of Molecular Oncology, Centre for DNA Fingerprinting and Diagnostics, Hyderabad, India; ${ }^{2}$ Department of \\ Histopathology, Apollo Hospitals, Hyderabad, India; ${ }^{3}$ Nizam's Institute of Medical Sciences, Hyderabad, India and \\ ${ }^{4}$ Department of Pathology, Stanford University School of Medicine, Stanford, CA, USA
}

Background: The human ATP-dependent SWItch/sucrose nonfermentable (SWI/SNF) complex functions as a primary chromatin remodeler during ontogeny, as well as in adult life. Several components of the complex have been suggested to function as important regulators of tumorigenesis in various cancers. In the current study, we have characterised a possible tumour suppressor role for the largest subunit of the complex, namely the AT-rich interaction domain 1B (ARID1B).

Methods: We performed Azacytidine and Trichostatin A treatments, followed by bisulphite sequencing to determine the possible DNA methylation-induced transcription repression of the gene in pancreatic cancer ( $\mathrm{PaCa}$ ) cell lines. Functional characterisation of effect of ARID1B ectopic expression in MiaPaCa2 PaCa cell line, which harboured ARID1B homozygous deletion, was carried out. Finally, we evaluated ARID1B protein expression in pancreatic tumour samples using immunohistochemistry on a tissue microarray.

Results: ARID1B was transcriptionally repressed due to promoter hypermethylation, and ectopic expression severely compromised the ability of MiaPaCa2 cells to form colonies in liquid culture and soft agar. In addition, ARID1B exhibited significantly reduced/loss of expression in $\mathrm{PaCa}$ tissue, especially in samples from advanced-stage tumours, when compared with normal pancreas.

Conclusion: The results therefore suggest a possible tumour-suppressor function for $A R I D 1 B$ in $\mathrm{PaCa}$, thus adding to the growing list of SWI/SNF components with a similar function. Given the urgent need to design efficient targeted therapies for $\mathrm{PaCa}$, our study assumes significance.

Pancreatic cancer $(\mathrm{PaCa})$ continues to be a devastating disease despite recent improvements in the understanding of its biology. Pancreatic tumours are frequently associated with post-resection recurrence and are refractory to available treatment options (Buchler et al, 1991). Most patients harbour distant metastasis at the time of presentation, and therefore not eligible for resection; the 5-year survival rate for such patients is close to nil. Identification of $\mathrm{PaCa}$ oncogenes exhibiting recurrent activation including KRAS and AKT2, and of tumour suppressor genes (TSGs) exhibiting recurrent inactivation including TP53, CDKN2A

\footnotetext{
*Correspondence: Dr MD Bashyam; E-mail: bashyam@cdfd.org.in

${ }^{5}$ These authors contributed equally to this work

${ }^{6}$ Present address: Krishna Institute of Medical Sciences, Hyderabad
}

Revised 8 March 2013; accepted 8 April 2013; published online 9 May 2013

(c) 2013 Cancer Research UK. All rights reserved 0007-0920/13 
and SMAD4 has improved our understanding of pancreatic tumours (Bardeesy and DePinho, 2002). Recent advances in the field of cancer genomics have revealed interesting insights into the complex biology of the disease and are expected to hasten the development of efficient targeted therapies. Given the ease of targeting, researchers worldwide have focused their efforts on characterisation of $\mathrm{PaCa}$ oncogenes; the study of PaCa TSGs has been neglected to a significant extent. Of note, recent studies have indicated that a majority of driver mutations in all cancers occur in TSGs rather than oncogenes (Bozic et al, 2010).

Epigenetic modifications are cardinal regulators of eukaryotic gene expression, especially with respect to embryonic development and oncogenic transformation. The human SWItch/sucrose nonfermentable (SWI/SNF) ATP-dependent chromatin-remodelling complex, components of which are orthologs of the Drosophila trithorax family (Kennison, 1995; Schuettengruber et al, 2007), includes either of the two ATPase-containing subunits, namely BRG1 and BRM. The largest subunit named BAF250 or AT-rich interaction domain 1 (ARID1) exists in two mutually exclusive isoforms viz BAF250a (ARID1A) and BAF250b (ARID1B) (Wang et al, 1996). Based on presence or absence of these subunits, four distinct complexes can be envisaged. In addition, another variant (the PBAF complex) has been reported, which harbours BRG1 in association with BAF180 and BAF200 (Yan et al, 2005). Mutation/deletion-induced inactivation of BRG1 and, to a lesser extent, of BRM has been described in many tumour types (Weissman and Knudsen, 2009). Recently, inactivation of SNF5 (a core component of the complex), resulting in alleviation of repression of Polycomb group proteins such as EZH2, was shown to be an important driver event in malignant rhabdoid tumours (Wilson et al, 2010). In addition, tumour-suppressor roles for BAF155 and BAF57 (core components of the complex) have also been validated (Weissman and Knudsen, 2009).

Our earlier studies based on array-based comparative genomic hybridisation (aCGH) carried out on PaCa cell lines (Bashyam et al, 2005) and xenografts (Kwei et al, 2008) led to the identification of several localised amplifications and deletions in specific chromosomal regions. Characterisation of two recurrent amplifications at $18 \mathrm{q} 11.2$ and $7 \mathrm{q} 21$ led to the assignment of an oncogenic function to GATA6 (Kwei et al, 2008) and SMURF1 (Kwei et al, 2011), respectively, in PaCa. More importantly, we identified several localised homozygous deletions validated by multiplex PCR, including one located at 6q25.3 that harboured a single annotated gene viz ARID1B (Bashyam et al, 2005). In the current study, we propose a tumour-suppressor function for $A R I D 1 B$ in $\mathrm{PaCa}$ based on functional studies, methylation analysis of promoter $\mathrm{CpG}$ islands and expression analysis in pancreas tumour samples.

\section{MATERIALS AND METHODS}

PaCa cell line propagation and manipulation. $\mathrm{PaCa}$ cell lines were procured from the ATCC, USA; propagation and DNA/RNA isolations were performed as described earlier (Bashyam et al, 2005). For Azacytidine and Trichostatin A (TSA) (Sigma, St. Louis, MO, USA) treatments, 0.2 million cells were seeded in $60-\mathrm{mm}$ dishes and exposed (amounts (in $\mu \mathrm{M}$ ) indicated in Figures $1 \mathrm{~A}$ and $\mathrm{B}$ ) for $24 \mathrm{~h}$, followed by a fresh exposure for $24 \mathrm{~h}$. Quantitative reverse transcription PCR was performed to evaluate ARID1B transcript levels relative to GAPDH as described in Supplementary Methods S1.

Analysis of ARID1B CpG methylation. Putative CpG island was identified upstream of the ARID1B start codon using MethPrimer (www.urogene.org/methprimer/index1.html) and CPGPLOT (www.ebi.ac.uk/Tools/emboss/cpgplot) with default parameters.
PCR primer pairs specific to modified DNA sequence and flanking the putative ARID1B CpG-rich sequence were designed using MethPrimer such that each amplicon size was $<400$ bp (Figure 1C top panel and Supplementary Methods S1). All primers (except 3PR) did not include $\mathrm{CpG}$ dinucleotide (sequences are listed in Supplementary Methods S1). Bisulphite modification of genomic DNA isolated from cell lines was performed using EZ DNA Methylation-Gold Kit (Zymo Research, Irvine, CA, USA), as per manufacturer's instructions. PCR products were cloned into the pCR 2.1-TOPO vector (Invitrogen, Carlsbad, CA, USA), as per standard protocol. Recombinant plasmids were sequenced using the $3130 \times 1$ genetic analyser (ABI Inc, Foster city, CA, USA), as per the manufacturer's instructions. A conversion frequency of $>98 \%$ was confirmed in each recombinant plasmid.

Ectopic expression of ARID1B and functional assays. ARID1B cDNA construct (KIAA1235), a kind gift from the Kazusa DNA research institute (Chiba, Japan), encodes a partially truncated 1485 amino acid protein (devoid of the N-terminal 805 amino acids). Previous studies have validated functional activity of the ARID1B protein expressed from this construct (Inoue et al, 2002, 2011). ARID1B cDNA was cloned into the pcDNA3.1HisC vector as described in Supplementary Methods S1. MiaPaCa2 cells were transfected with the ARID1B-pcDNA3.1HisC recombinant plasmid construct in 60-mm culture dishes using lipofectamine 2000 (Invitrogen), as per manufacturer's protocol; pcDNA31.HisC vector was transfected separately as a control. Transfected cells were passaged into $100-\mathrm{mm}$ dishes in presence of $100 \mu \mathrm{g} \mathrm{ml}^{-1}$ Neomycin (G418) to obtain a single cell spread. Neomycinresistant colonies appeared after 20-21 days of selection and were isolated by localised trypsinization method, grown separately on 96-well plates and further expanded in 6-well plates. Expression of the Neomycin gene in recombinant clones was confirmed by RT-PCR (data not shown). ARID1B expression was confirmed using RT-PCR in the ARID1B clones (but not in the vector clones), as per strategy outlined in Supplementary Methods S1. In separate transfection experiments, colonies (obtained from both $A R I D 1 B$ and vector transfectants of MiaPaCa2 and PANC1 cells) were pooled independently following 21 days of selection under Neomycin to perform a colony-formation assay (Supplementary Methods S1). Other functional assays were performed as described in Supplementary Methods S1.

Construction of tissue microarray (TMA) and immunohistochemistry (IHC). A PaCa TMA was constructed using the Minicore tissue arrayer (Alphelys, Plaisir, France) as described previously (Ginestier et al, 2002). A total of 52 sample pairs (tumour and matched normal) in the form of formalin-fixed paraffin-embedded blocks representing pancreatic tumours were collected from the Nizam's Institute of Medical Sciences, Hyderabad, following approval from the hospital ethics committee. The samples included well, moderate and poorly differentiated ductal adenocarcinoma; specialized adenocarcinoma including cyst-adenocarcinoma, mucinous and papillary adenocarcinoma and clear cell carcinoma; and neuroendocrine tumours. The array was constructed with at least two representative 1 -mm cores from each tumour and normal sample. IHC was performed on $4-\mu \mathrm{m}$ sections on an automated immunostainer (Ventana Benchmark $\mathrm{XT}$, Tucson, AZ, USA) using ARID1B (clone 2F2, Novus Biologicals, Littleton, CO, USA; dilution of $1: 200$ ) and p53 (clone DO-1, EMD Millipore Calbiochem, Billerica, MA, USA; dilution of $1: 100$ ) antibodies separately, as per manufacturer's instructions. For ARID1B, nuclear staining intensity (absent, weak, moderate and strong; 0-3 scale) and fractional epithelium staining (absent, up to $25,50,75$ and $100 \%$; $0-4$ scale) were evaluated by the pathologist and summated for a final staining score. For p53, $>20 \%$ positivity was considered as nuclear stabilisation. 

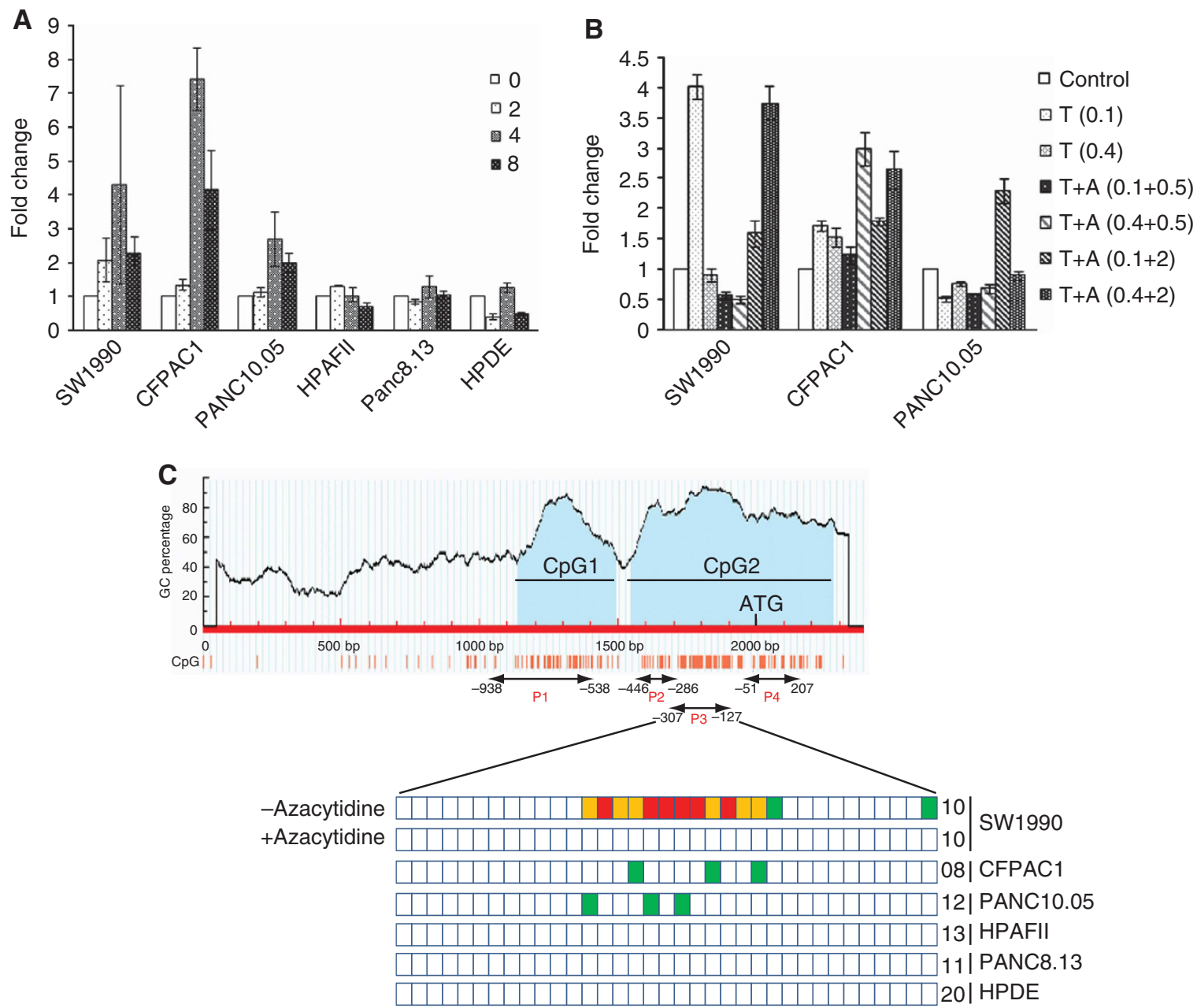

Figure 1. Evaluation of CPG methylation-induced transcriptional repression of ARID1B. Panels (A) and (B) show effect of Azacytidine and TSA, respectively, on several $\mathrm{PaCa}$ cell lines; fold increase in ARID1B transcript level in each cell line is plotted separately in response to varying concentrations (in $\mu \mathrm{m}$ indicated) of Azacytidine and TSA. The transcript level in absence of treatment in each cell line is normalised to 1.0. Error bars represent s.e.m.; each Azacytidine/TSA experiment was performed at least thrice. A, Azacytidine; T, Trichostatin (A). Panel (C) (top) shows position of the four ARID1B primer pairs used for PCR amplification of putative ARID1B promoter CpG island. Nucleotide position of the $5^{\prime}$ and $3^{\prime}$ ends of the four amplicons with respect to the translation initiation codon (ATG) are indicated. The bottom panel depicts the result of bisulphite sequencing-based evaluation of methylation status for the P3 region. Each row of boxes represents result for one cell line; each box represents one CpG dinucleotide and per cent of cytosine methylation is denoted by a colour code (white, <10\%; green, 10-33\%; orange, 34-66\%; red, $>66 \%$ ). The total number of clones analysed for each cell line is given at the end of each row of boxes. Methylation status in SW1990 was also evaluated, following Azacytidine $(8 \mu \mathrm{M})$ treatment.

\section{RESULTS}

ARID1B exhibits promoter CpG methylation-induced transcriptional repression in $\mathrm{PaCa}$ cell lines. TSGs are inactivated in tumour cells through several distinct mechanism(s), not the least being mutation and methylation-induced transcription silencing. We screened genomic DNA isolated from $10 \mathrm{PaCa}$ cell lines for presence of mutations in the coding region, promoter, UTRs and splice consensus sequences by PCR-DNA sequencing and did not detect a single mutation (data not shown). In addition, the same $A R I D 1 B$ transcript isoform was detected in $\mathrm{PaCa}$ cell lines and pancreatic normal tissue (data not shown). Azacytidine, frequently used to determine genes transcriptionally repressed through methylated CpGs in the promoter, caused a significant elevation in ARID1B transcript levels in SW1990, PANC10.05 and CFPAC1, but not in PANC8.13, HPAFII and PANC1, commensurate with lower (SW1990, PANC10.05 and CFPAC1; Supplementary Figure S1) and higher (PANC8.13, HPAFII and PANC1; Supplementary Figure S1) ARID1B transcript levels (Figure 1A).
In addition, Azacytidine had no significant effect on ARID1B transcript level in the nontumorigenic human pancreatic ductal epithelial (HPDE) cells (Figure 1A) known to exhibit adequate expression of SWI/SNF components, including ARID1B (Ouyang et al, 2000; Shain et al, 2012). Elevation in ARID1B transcript levels was evident in SW1990, PANC10.05 and CFPAC1 when subjected to TSA treatment as well (Figure 1B).

Next, we identified CpG-rich sequences in the putative promoter region of $A R I D 1 B$, spanning about $2 \mathrm{~kb}$ upstream of the translation initiation codon (Figure 1C top panel). Bisulphite sequencing using primer pairs specific for the CpG-rich regions (Figure 1C and Supplementary Methods S1) revealed significant methylation of $\mathrm{CpGs}$ located in the $\mathrm{P} 3$ region (present immediately upstream to the ARIDIB translation initiation codon; Figure 1C top panel), particularly in SW1990. Further, the methylation (in SW1990) was completely alleviated upon Azacytidine treatment (Figure 1C, bottom panel), suggesting that methylation was responsible for $A R I D 1 B$ transcription silencing. Moderately significant methylation in $\mathrm{P} 2$ and $\mathrm{P} 4$ regions was also observed (Supplementary Figure S2), though no significant methylation was 

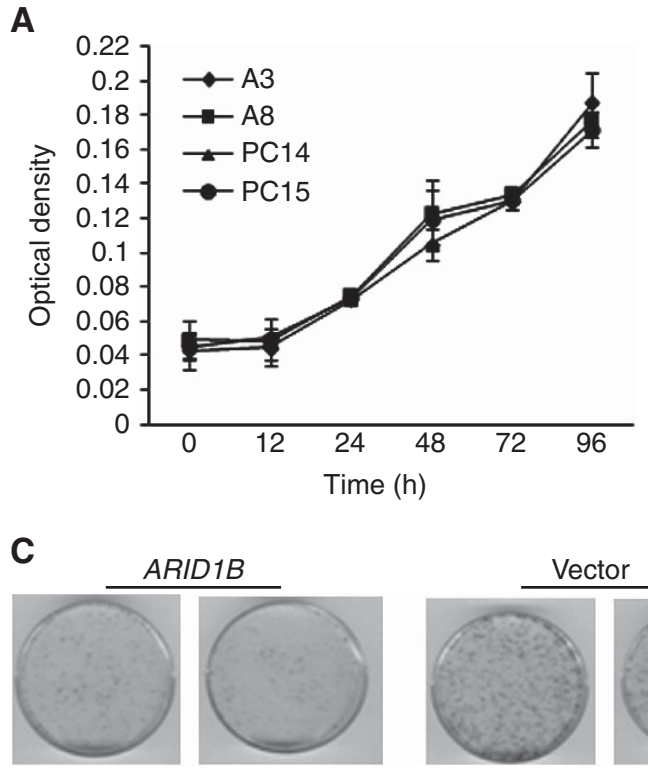

A3

A8

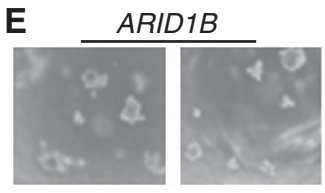

A3

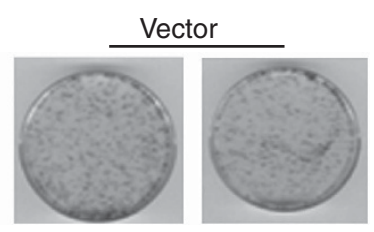

PC14

PC15
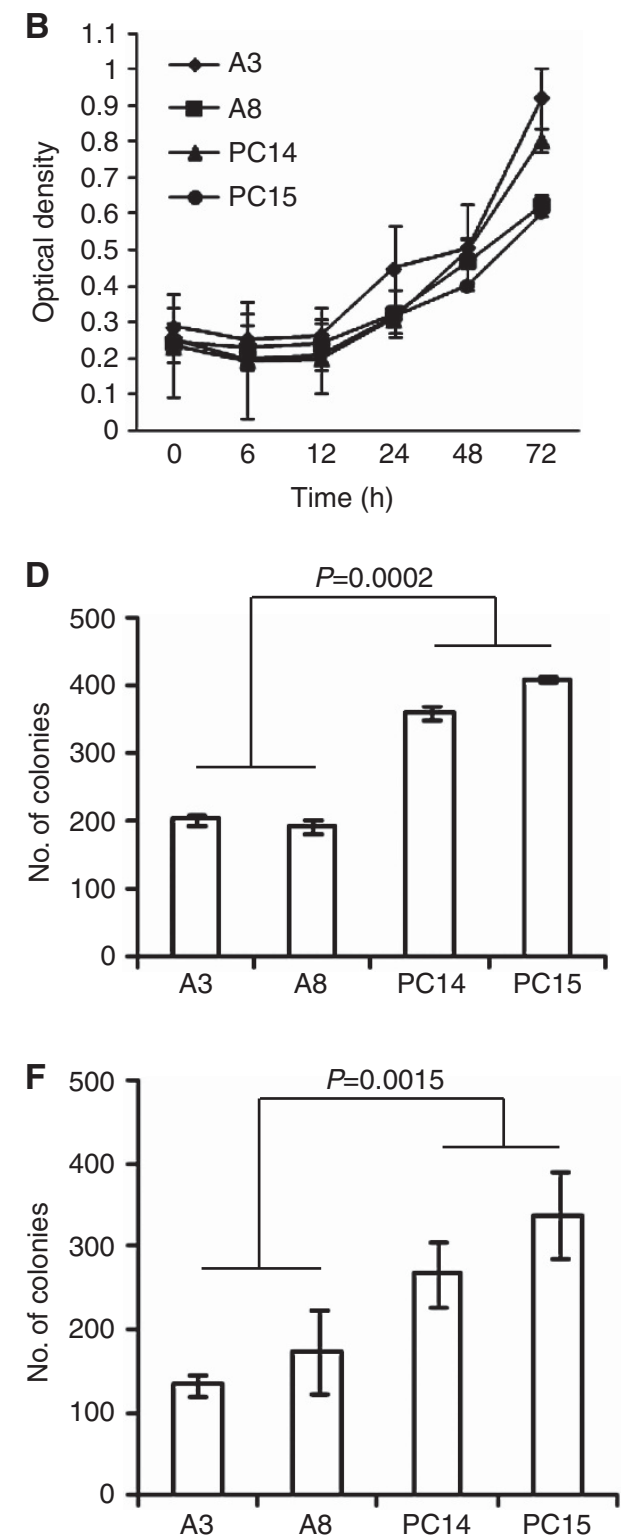

Figure 2. Functional characterisation of ARID1B permanent transfectants of MiaPaCa2. Panels (A) and (B) show result of MTT and crystal violet dye extraction growth assays performed for the two ARID1B (A3 and A8) and two vector (PC14 and PC15) clones of MiaPaCa2, respectively. Error bars represent s.e.m. of two (MTT assay) and three (crystal violet assay) independent experiments (performed in duplicate), respectively. Panels (C) and (E) show representative results of liquid and soft agar colony-formation assays, respectively. Panels (D) and (F) show graphical representation of result of liquid and soft agar colony-formation assays, respectively. Panel (G) shows quantitation of $\beta$-galactosidase staining in the same clones. Error bars represent s.e.m. of three independent liquid colony formation (in duplicate), soft agar and $\beta$-galactosidase staining (both in triplicate) experiments. P-value corresponds to unpaired Student's t-test.

detected in the P1 region (data not shown). In contrast, PANC8.13, HPAFII and HPDE either harboured negligible or nil methylation of the CpGs in these regions (Figure $1 \mathrm{C}$ and Supplementary Figure S2).

Ectopic expression of ARID1B abrogates tumour-related characteristics of $\mathrm{PaCa}$ cell line. In our previous study, the 6q25.3 homozygous deletion (harbouring the ARID1B gene) identified in the MiaPaCa2 $\mathrm{PaCa}$ cell line was PCR validated using primer pairs specific for the $A R I D 1 B$ 3'-UTR region (Bashyam et al, 2005). Therefore, we first confirmed ARID1B complete deletion using PCR primers specific for exons 2, 5, 10, 12, 15,17 and 20 (data not shown). Next, in order to determine the effect of $A R I D 1 B$ ectopic expression in MiaPaCa2 cells, we generated pcDNA3.1HisC clones of ARID1B (and of the vector as a control) as described in the MATERIALS AND METHODS section. We chose two vector and $A R I D 1 B$ clones each for analysis. Expression of $A R I D 1 B$ transcript in the ARID1B clones was confirmed using RT-PCR (Supplementary Figure S3); however, protein expression could not be detected, though three different antibodies were tested (data not shown). MTT assay (Figure 2A), crystal violet dye extraction growth assay (Figure 2B), a standard plating assay (percentage of cells adhering to the surface upon seeding; data not shown) as well as FACS-based cell cycle analysis (data not shown) did not reveal a significant difference in growth kinetics of $A R I D 1 B$ and vector clones. Similarly, the percentage of apoptotic population in the gene and vector clones was not significantly different (data not shown). However, the $A R I D 1 B$ clones exhibited significantly reduced ability to form colonies in liquid culture as compared with the vector clones (Figures $2 \mathrm{C}$ and $\mathrm{D}$ ), indicating a reduced clonogenicity in case of the former. In addition, the $A R I D 1 B$ clones generated significantly 

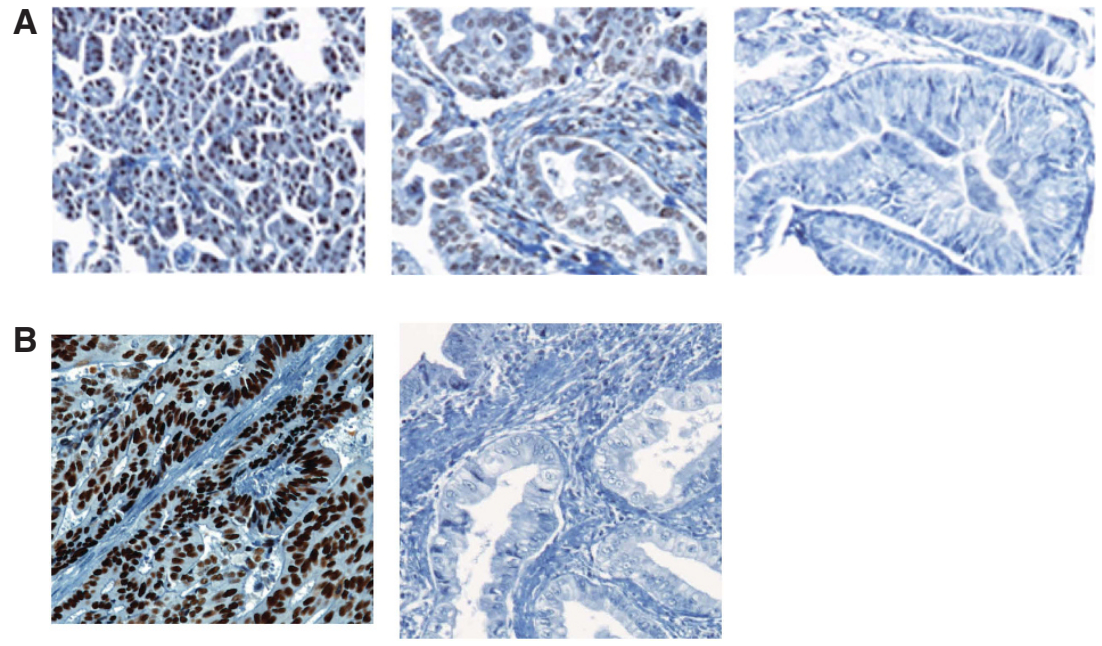

C

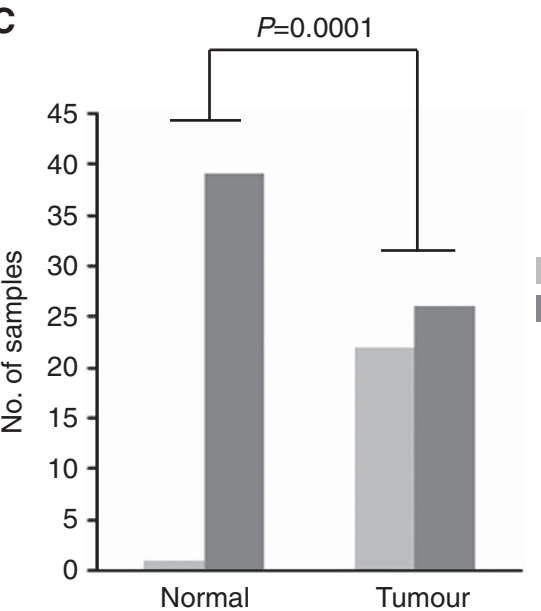

D

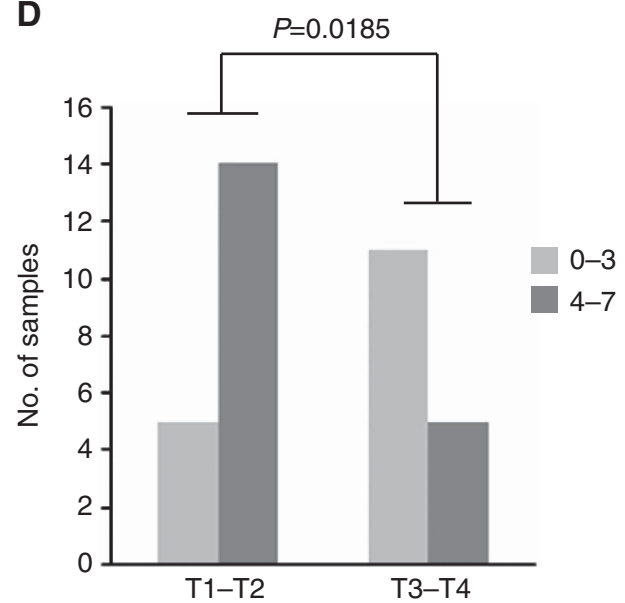

Figure 3. Analysis of ARID1B and p53 expression using a pancreatic cancer tissue microarray. Panel (A) shows representative results for ARID1B for normal pancreas (left, strong nuclear stain) and pancreatic tumour (middle, moderate nuclear stain and right, negative nuclear stain). Representative results for p53 IHC performed on PaCa samples are shown in panel (B) (left, positive; right, negative). Panel (C) shows graphical representation of comparative analysis of ARID1B expression in pancreatic tumour and normal samples; samples were split into two categories based on their IHC scores (0-3 representing negative to weak staining and 4-7, indicating moderate to strong staining) calculated as described in MATERIALS AND METHODS. Panel (D) shows graphical representation of comparative analysis of ARID1B expression in early (T1-T2) and late (T3-T4) stage pancreatic tumour samples, based on IHC scores. Fisher's exact test $P$-values are shown.

less number (and smaller size) of colonies in soft agar, indicating a reduced ability to grow in the absence of anchorage (Figures $2 \mathrm{E}$ and F). In order to rule out the possibility of reduced colonyformation ability of $A R I D 1 B$ clones being attributable specifically to the two selected clones and not to $A R I D 1 B$ expression, we performed liquid colony-formation assay separately using a 'pool' of $A R I D 1 B$ and vector clones obtained from an independent MiaPaCa2 transfection experiment (MATERIALS AND METHODS). The pooled ARID1B clones generated significantly fewer colonies compared with the pooled vector clones, whereas there was no significant difference in colonies generated from pooled vector and $A R I D 1 B$ PANC1 clones (Supplementary Figure S4), thus strengthening our premise that $A R I D 1 B$ expression was indeed responsible for reduced colony-formation ability. Given that ectopic expression of the SWI/SNF component BRG1 induces a senescence phenotype in rat mesenchymal stem cells (Napolitano et al, 2007) as well as in PaCa cells (Shain et al, 2012), we tested whether $A R I D 1 B$ expression could have a similar effect on $\mathrm{MiaPaCa} 2$ cells. The ARID1B clones exhibited significantly higher senescence-associated $\beta$-galactosidase activity when compared with the vector clones (Supplementary Figure S5 and Figure $2 \mathrm{G}$ ).
Reduced/loss of ARID1B expression in PaCa tissues. We constructed a TMA to evaluate ARID1B and p53 expression status in $\mathrm{PaCa}$ tumour samples using IHC (Figures $3 \mathrm{~A}$ and $\mathrm{B}$ ). Interestingly, a significant proportion of tumour samples exhibited negative/reduced staining when compared with normal pancreas $(P=0.0001$, Fisher's exact test; Figure 3C). Moreover, the loss of ARID1B expression was significantly associated with advancedtumour stage ( $P=0.0185$, Fisher's exact test; Figure 3D), indicating perhaps it could be a late event. As a comparison, p53 exhibited nuclear stabilisation in 19 of 42 samples (representative result shown in Figure 3B) and there was no association with ARID1B status nor with tumour stage (data not shown). These results provide further evidence for a tumour-suppressor role for $A R I D 1 B$ in $\mathrm{PaCa}$.

\section{DISCUSSION}

Deletion or rearrangement of the 6q25.3 region has been described in breast (Utada et al, 2000), cervical (Mazurenko et al, 2003) and 
other cancers (Monoranu et al, 2008). In the current study, we endeavoured to characterise a homozygous deletion localised at $6 \mathrm{q} 25.3$, identified in the $\mathrm{MiaPaCa} 2 \mathrm{PaCa}$ cell line in our previous studies (Bashyam et al, 2005; Shain et al, 2012) and in other studies (Birnbaum et al, 2011), which included only one annotated gene viz ARID1B. Gene mutation is a common mode of inactivation of TSGs; however, we did not detect a single mutation in $10 \mathrm{PaCa}$ cell lines tested. Azacytidine (Figure 1A) and Trichostatin A (Figure 1B) treatments, however, resulted in significant elevation in $A R I D 1 B$ transcript level in $\mathrm{PaCa}$ cell lines, suggesting suppression of gene expression through DNA methylation, which was confirmed by bisulphite sequencing (Figure 1C). HLTF, encoding another member of the SWI/SNF family, was earlier shown to be transcriptionally repressed following CpG methylation in lung cancer cell lines, and the repression was alleviated upon treatment with Azacytidine (Moinova et al, 2002).

Ectopic expression of SWI/SNF components into cancer cells has been shown to result in elevation of antitumor characteristics such as cell cycle arrest, apoptosis or differentiation, depending on the cancer cell type and the identity of the inactivated component (Caramel et al, 2008; Dunaief et al, 1994; Wang et al, 2005). Recent studies have suggested a tumour-suppressor role for components of the SWI/SNF complex in several cancers (Wilson and Roberts, 2011). SiRNA-mediated knockdown of $A R I D 1 B$ was earlier shown to cause a significant reduction of growth factor-independent growth in HPDE (Shain et al, 2012), further corroborating our observations. The reduced/loss of ARID1B expression in $\mathrm{PaCa}$ when compared with normal pancreatic tissues (Figure 3C) suggests the possible importance of the gene in human pancreatic tumours.

Previous studies on preosteoblast cell lines revealed opposing roles for ARID1A- and ARID1B-containing SWI/SNF complexes, with the former causing cell cycle arrest, whereas the latter appeared to be involved in cell cycle progression (Nagl et al, 2007). On the other hand, ARID1B levels increase during differentiation of embryonic stem cells (Kaeser et al, 2008) and ectopic expression induces increased expression of p53 and p21, followed by cell cycle arrest in HeLa cells (Inoue et al, 2011). It is therefore possible that ARID1B may have distinct roles depending on the cellular context. $A R I D 1 A$ appears to be a specific tumour suppressor for ovarian clear cell carcinomas (Wiegand et al, 2010). Interestingly, the inactivation of ARID1A in clear cell and endometrioid carcinomas also in breast cancer cell lines (Mamo et al, 2012) appears to be achieved predominantly through mutations, with little or no evidence of epigenetic inactivation (Wiegand et al, 2010). In contrast, we did not detect any ARID1B mutation in all $\mathrm{PaCa}$ cell lines screened. An earlier report had also revealed the absence of $A R I D 1 B$ mutations in $\mathrm{PaCa}$ cell lines and tumour samples (Birnbaum et al, 2011). The roles of ARID1A and ARID1B in different tumour types could possibly reflect differences in their tissue-specific expression.

We were unable to detect ARID1B protein expression in the permanent transfectants generated using pcDNA3.1HisC, though several clones were evaluated; the $A R I D 1 B$ transcript was however readily detected (Supplementary Figure S3). Tumour cells often exhibit reduced fitness when tumour suppressors are expressed at high levels (Kucuk et al, 2011). The phenotypic changes exhibited by the $A R I D 1 B-\mathrm{MiaPaCa} 2$ clones are probably a result of low-level expression that was undetected by western blot. As chromatin modulators are expected to generate a pleiotropic effect, a small increment in expression may be expected to result in visible phenotypic changes. A previous study on MiaPaCa2 cells (which lack expression of BRM) showed undetected BRM protein expression when overexpressed, but the low expression did result in phenotypic changes (Rosson et al, 2005). In the current study, overexpression of ARID1B had no significant effect on growth of $\mathrm{MiaPaCa} 2$ cells, though the transfectants exhibited significantly reduced ability to form colonies in liquid media and soft agar (Figures 2C-F). Of interest, the tumour suppressor ING4, which functions through interaction with chromatin remodelling factors, elicits a reduced clonogenicity without affecting proliferation in rat fibroblast cells (Kim et al, 2004). Similarly, ectopic expression of BAF155 (a core component of the human SWI/SNF complex) in cancer cell lines was recently shown to reduce colony-formation ability without affecting apoptosis (DelBove et al, 2011).

As MiaPaCa2 cells do not express BRM (Rosson et al, 2005), our observations pertaining to the antioncogenic effect of ARID1B expression is expected to be a result of the action of a SWI/SNF complex harbouring BRG1 and ARID1B. Interestingly, BRG1 and ARID1B have been suggested to harbour a similar expression profile and often localise to transcriptionally active promoters (Flores-Alcantar et al, 2011), as against BRM and ARID1A. Given that the BRG1-containing complex is known to activate genes that participate in cell cycle arrest (Napolitano et al, 2007) downstream of TGF beta signalling (Xi et al, 2008), and that the BRG1/ARID1B SWI/SNF complex activates expression of TSGs, such as p21 (Inoue et al, 2011), our results showing an increased senescenceassociated $\beta$-galactosidase activity in cells expressing ARID1B (Supplementary Figure S5 and Figure 2G) assume significance. The results obtained in this study therefore propose a tumour-suppressor role for ARID1B in PaCa.

\section{ACKNOWLEDGEMENTS}

The work was supported by grants (SR/SO/BB-49/2005 from the Department of Science and Technology, Government of India and BT/PR14407/BRB/10/833/2010 from the Department of Biotechnology, Government of India) to MDB, a core grant from the Department of Biotechnology, Government of India to MDB's host institute and an NIH grant (CA112016) to JRP. Md Khursheed, a registered PhD student of Manipal University, India, is grateful to the Council of Scientific and Industrial Research, Government of India, for a junior and senior research fellowship. We are grateful to the Kazusa DNA Research Centre, Japan, for kindly providing the ARID1B cDNA clone.

\section{REFERENCES}

Bardeesy N, DePinho RA (2002) Pancreatic cancer biology and genetics. Nat Rev Cancer 2: 897-909.

Bashyam MD, Bair R, Kim YH, Wang P, Hernandez-Boussard T, Karikari CA, Tibshirani R, Maitra A, Pollack JR (2005) Array-based comparative genomic hybridisation identifies localised DNA amplifications and homozygous deletions in pancreatic cancer. Neoplasia 7: 556-562.

Birnbaum DJ, Adelaide J, Mamessier E, Finetti P, Lagarde A, Monges G, Viret F, Goncalves A, Turrini O, Delpero JR, Iovanna J, Giovannini M, Birnbaum D, Chaffanet M (2011) Genome profiling of pancreatic adenocarcinoma. Genes Chromosomes Cancer 50: 456-465.

Bozic I, Antal T, Ohtsuki H, Carter H, Kim D, Chen S, Karchin R, Kinzler KW, Vogelstein B, Nowak MA (2010) Accumulation of driver and passenger mutations during tumour progression. Proc Natl Acad Sci USA 107: 18545-18550.

Buchler M, Friess H, Schultheiss KH, Gebhardt C, Kubel R, Muhrer KH, Winkelmann M, Wagener T, Klapdor R, Kaul M, Müller G, Schulz G, Beger HG (1991) A randomized controlled trial of adjuvant immunotherapy (murine monoclonal antibody 494/32) in resectable pancreatic cancer. Cancer 68: 1507-1512.

Caramel J, Medjkane S, Quignon F, Delattre O (2008) The requirement for SNF5/INI1 in adipocyte differentiation highlights new features of malignant rhabdoid tumours. Oncogene 27: 2035-2044.

DelBove J, Rosson G, Strobeck M, Chen J, Archer TK, Wang W, Knudsen ES, Weissman BE (2011) Identification of a core member of the SWI/SNF complex, BAF155/SMARCC1, as a human tumour suppressor gene. Epigenetics 6: 1444-1453. 
Dunaief JL, Strober BE, Guha S, Khavari PA, Alin K, Luban J, Begemann M, Crabtree GR, Goff SP (1994) The retinoblastoma protein and BRG1 form a complex and cooperate to induce cell cycle arrest. Cell 79: 119-130.

Flores-Alcantar A, Gonzalez-Sandoval A, Escalante-Alcalde D, Lomeli H (2011) Dynamics of expression of ARID1A and ARID1B subunits in mouse embryos and in cells during the cell cycle. Cell Tissue Res 345: 137-148.

Ginestier C, Charafe-Jauffret E, Bertucci F, Eisinger F, Geneix J, Bechlian D, Conte N, Adelaide J, Toiron Y, Nguyen C, Viens P, Mozziconacci MJ, Houlgatte R, Birnbaum D, Jacquemier J (2002) Distinct and complementary information provided by use of tissue and DNA microarrays in the study of breast tumour markers. Am J Pathol 161: 1223-1233.

Inoue H, Furukawa T, Giannakopoulos S, Zhou S, King DS, Tanese N (2002) Largest subunits of the human SWI/SNF chromatin-remodelling complex promote transcriptional activation by steroid hormone receptors. J Biol Chem 277: 41674-41685.

Inoue $\mathrm{H}$, Giannakopoulos $\mathrm{S}$, Parkhurst $\mathrm{CN}$, Matsumura T, Kono EA, Furukawa T, Tanese N (2011) Target genes of the largest human SWI/SNF complex subunit control cell growth. Biochem J 434: 83-92.

Kaeser MD, Aslanian A, Dong MQ, Yates JR, Emerson BM (2008) BRD7, a novel PBAF-specific SWI/SNF subunit, is required for target gene activation and repression in embryonic stem cells. J Biol Chem 283: 32254-32263.

Kennison JA (1995) The Polycomb and trithorax group proteins of Drosophila: trans-regulators of homeotic gene function. Annu Rev Genet 29: 289-303.

Kim S, Chin K, Gray JW, Bishop JM (2004) A screen for genes that suppress loss of contact inhibition: identification of ING4 as a candidate tumour suppressor gene in human cancer. Proc Natl Acad Sci USA 101: 16251-16256.

Kucuk C, Iqbal J, Hu X, Gaulard P, De Leval L, Srivistava G, Au WY, McKeithan TW, Chan WC (2011) PRDM1 is a tumor suppressor gene in natural killer cell malignancies. Proc Natl Acad Sci USA 108: 20119-20124.

Kwei KA, Bashyam MD, Kao J, Ratheesh R, Reddy EC, Kim YH, Montgomery K, Giacomini CP, Choi YL, Chatterjee S, Karikari CA, Salari K, Wang P, Hernandez-Boussard T, Swarnalata G, van de Rijn M, Maitra A, Pollack JR (2008) Genomic profiling identifies GATA6 as a candidate oncogene amplified in pancreatobiliary cancer. PLoS Genet 4: e1000081.

Kwei KA, Shain AH, Bair R, Montgomery K, Karikari CA, van de Rijn M, Hidalgo M, Maitra A, Bashyam MD, Pollack JR (2011) SMURF1 amplification promotes invasiveness in pancreatic cancer. PLoS One 6: e23924.

Mamo A, Cavallone L, Tuzmen S, Chabot C, Ferrario C, Hassan S, Edgren H, Kallioniemi O, Aleynikova O, Przybytkowski E, Malcolm K, Mousses S, Tonin PN, Basik M (2012) An integrated genomic approach identifies ARID1A as a candidate tumour-suppressor gene in breast cancer. Oncogene 31: 2090-2100.

Mazurenko NN, Beliakov IS, Bliev A, Guo Z, Hu X, Vinokurova SV, Bidzhieva BA, Pavlova LS, Ponten J, Kiselev FL (2003) [Cervical carcinoma progression-associated genetic alterations on chromosome 6]. Mol Biol (Mosk) 37: 472-481.

Moinova HR, Chen WD, Shen L, Smiraglia D, Olechnowicz J, Ravi L, Kasturi L, Myeroff L, Plass C, Parsons R, Minna J, Willson JK, Green SB, Issa JP, Markowitz SD (2002) HLTF gene silencing in human colon cancer. Proc Natl Acad Sci USA 99: 4562-4567.

Monoranu CM, Huang B, Zangen IL, Rutkowski S, Vince GH, Gerber NU, Puppe B, Roggendorf W (2008) Correlation between 6q25.3 deletion status and survival in pediatric intracranial ependymomas. Cancer Genet Cytogenet 182: 18-26.

Nagl Jr. NG, Wang X, Patsialou A, Van Scoy M, Moran E (2007) Distinct mammalian SWI/SNF chromatin remodelling complexes with opposing roles in cell-cycle control. Embo J 26: 752-763.
Napolitano MA, Cipollaro M, Cascino A, Melone MA, Giordano A, Galderisi U (2007) Brgl chromatin remodelling factor is involved in cell growth arrest, apoptosis and senescence of rat mesenchymal stem cells. J Cell Sci 120: 2904-2911.

Ouyang H, Mou L, Luk C, Liu N, Karaskova J, Squire J, Tsao MS (2000) Immortal human pancreatic duct epithelial cell lines with near normal genotype and phenotype. Am J Pathol 157: 1623-1631.

Rosson GB, Bartlett C, Reed W, Weissman BE (2005) BRG1 loss in $\mathrm{MiaPaCa} 2$ cells induces an altered cellular morphology and disruption in the organization of the actin cytoskeleton. J Cell Physiol 205: 286-294.

Schuettengruber B, Chourrout D, Vervoort M, Leblanc B, Cavalli G (2007) Genome regulation by polycomb and trithorax proteins. Cell $\mathbf{1 2 8}$ : 735-745.

Shain AH, Giacomini CP, Matsukuma K, Karikari CA, Bashyam MD, Hidalgo M, Maitra A, Pollack JR (2012) Convergent structural alterations define SWItch/Sucrose NonFermentable (SWI/SNF) chromatin remodeler as a central tumour suppressive complex in pancreatic cancer. Proc Natl Acad Sci USA 109: E252-E259.

Utada Y, Haga S, Kajiwara T, Kasumi F, Sakamoto G, Nakamura Y, Emi M (2000) Allelic loss at the 8p22 region as a prognostic factor in large and estrogen receptor negative breast carcinomas. Cancer $\mathbf{8 8}$ : $1410-1416$.

Wang L, Baiocchi RA, Pal S, Mosialos G, Caligiuri M, Sif S (2005) The BRG1and hBRM-associated factor BAF57 induces apoptosis by stimulating expression of the cylindromatosis tumour suppressor gene. Mol Cell Biol 25: 7953-7965.

Wang W, Xue Y, Zhou S, Kuo A, Cairns BR, Crabtree GR (1996) Diversity and specialization of mammalian SWI/SNF complexes. Genes Dev 10: 2117-2130.

Weissman B, Knudsen KE (2009) Hijacking the chromatin remodelling machinery: impact of SWI/SNF perturbations in cancer. Cancer Res 69: 8223-8230.

Wiegand KC, Shah SP, Al-Agha OM, Zhao Y, Tse K, Zeng T, Senz J, McConechy MK, Anglesio MS, Kalloger SE, Yang W, Heravi-Moussavi A, Giuliany R, Chow C, Fee J, Zayed A, Prentice L, Melnyk N, Turashvili G, Delaney AD, Madore J, Yip S, McPherson AW, Ha G, Bell L, Fereday S, Tam A, Galletta L, Tonin PN, Provencher D, Miller D, Jones SJ, Moore RA, Morin GB, Oloumi A, Boyd N, Aparicio SA, Shih Ie,M, Mes-Masson AM, Bowtell DD, Hirst M, Gilks B, Marra MA, Huntsman DG (2010) ARID1A mutations in endometriosis-associated ovarian carcinomas. N Engl J Med 363: 1532-1543.

Wilson BG, Roberts CW (2011) SWI/SNF nucleosome remodellers and cancer. Nat Rev Cancer 11: 481-492.

Wilson BG, Wang X, Shen X, McKenna ES, Lemieux ME, Cho YJ, Koellhoffer EC, Pomeroy SL, Orkin SH, Roberts CW (2010) Epigenetic antagonism between polycomb and SWI/SNF complexes during oncogenic transformation. Cancer Cell 18: 316-328.

Xi Q, He W, Zhang XH, Le HV, Massague J (2008) Genome-wide impact of the BRG1 SWI/SNF chromatin remodeler on the transforming growth factor beta transcriptional program. J Biol Chem 283: 1146-1155.

Yan Z, Cui K, Murray DM, Ling C, Xue Y, Gerstein A, Parsons R, Zhao K, Wang W (2005) PBAF chromatin-remodelling complex requires a novel specificity subunit, BAF200, to regulate expression of selective interferonresponsive genes. Genes Dev 19: 1662-1667.

This work is published under the standard license to publish agreement. After 12 months the work will become freely available and the license terms will switch to a Creative Commons AttributionNonCommercial-Share Alike 3.0 Unported License.

Supplementary Information accompanies this paper on British Journal of Cancer website (http://www.nature.com/bjc) 